\title{
AVAglio: Phase 3 Trial of Bevacizumab Plus Temozolomide and Radiotherapy in Newly Diagnosed Glioblastoma Multiforme
}

\author{
O. L. Chinot · T. de La Motte Rouge · N. Moore · A. Zeaiter · A. Das · H. Phillips · Z. Modrusan · T. Cloughesy
}

Received: August 4, 2010 / Published online: March 14, 2011

(c) The Author(s) 2011. This article is published with open access at Springerlink.com

\section{ABSTRACT}

Despite treatment with the current standardof-care therapies, patients with newly diagnosed glioblastoma multiforme (GBM) exhibit dismal prognoses. Bevacizumab has demonstrated activity in patients with recurrent GBM and phase 2 trials indicate that the combination of bevacizumab with standard-of-care therapy is feasible and active for patients with newly diagnosed GBM. Bevacizumab has been granted US approval for use as single-agent therapy for patients with progressive GBM following prior

\section{O. L. Chinot $(\bowtie)$}

Service de Neuro-Oncologie, Centre Hospitalo-

Universitaire Timone, Université de la Méditerranée,

264 Rue Saint Pierre, 13385 Marseilles Cedex 05, France.

Email: olivier.chinot@ap-hm.fr

T. de La Motte Rouge

Service d'Oncologie Médicale, Groupe Hospitalier Pitié

Salpétrière, Paris, France

N. Moore $\cdot$ A. Zeaiter

F. Hoffmann-La Roche Ltd., Basel, Switzerland

A. Das · H. Phillips · Z. Modrusan

Genentech, Inc., San Francisco, CA, USA

T. Cloughesy

David Geffen School of Medicine, University of

California, Los Angeles, CA, USA therapy, although it has not received approval for use in patients with GBM in Europe. Phase 3 studies have been initiated in patients with newly diagnosed GBM and are currently recruiting patients. We describe the protocol for the AVAglio phase 3 registration trial, which is designed to evaluate the efficacy and safety of combining bevacizumab with standard-of-care therapy in patients with newly diagnosed GBM.

Keywords: bevacizumab; clinical trial; glioblastoma multiforme; phase 3

\section{INTRODUCTION}

Population-based studies report a poor prognosis for patients with newly diagnosed glioblastoma multiforme (GBM), with 1-year, 2 -year, and 5-year survival rates at $17 \%$ to $30 \%$, $2 \%$ to $8 \%$, and $1 \%$ to $3 \%$, respectively. ${ }^{1,2}$ Despite recent advances in imaging techniques, surgery, and medical therapy, ${ }^{1}$ prognosis remains dismal. The current standard-of-care therapy (radiotherapy plus temozolomide followed by six cycles of adjuvant temozolomide) provided 2 -year and 5-year survival rates of $27 \%$ and $10 \%$ for patients with newly diagnosed GBM in a clinical trial setting. ${ }^{3}$ While this indicates an 
important improvement in survival benefit, a clear unmet clinical need still remains.

Bevacizumab (Avastin ${ }^{\circledR}$, Genentech, Inc., San Francisco, CA, USA), a humanized monoclonal antibody that binds to and inhibits the activity of vascular endothelial growth factor (VEGF), acts with cytotoxic chemotherapy or biological agents in the treatment of various tumors, eg, colorectal, lung, renal, and breast cancer. ${ }^{4-7}$ GBM is characterized by sustained angiogenesis; the key regulator of tumor angiogenesis is VEGF, which is frequently overexpressed in $\mathrm{GBM}^{8,9}$ as well as other tumor types (Figure 1). Bevacizumab inhibits $\operatorname{VEGF}^{10}$ and has been shown in preclinical models to have activity against GBM both alone and in combination with radiotherapy and temozolomide. ${ }^{11,12}$ Bevacizumab has also shown considerable potential to provide clinical benefit for patients with GBM (see below).

\section{BEVACIZUMAB TRIAL DATA IN GBM}

Various exploratory noncomparative clinical trials provided initial data suggesting that bevacizumab alone or in combination with irinotecan is active and has a manageable safety profile in patients with recurrent GBM. ${ }^{13,14}$ This benefit and safety profile was confirmed in a randomized, noncomparative phase 2

Figure 1. Vascular endothelial growth factor mRNA overexpression by various tumor types detected on Affymetrix U133P microarray after staining in Affymetrix Fluidics station and scanning on GeneChip scanner 300 (data on file; Genentech, Inc., San Francisco, CA, USA). GBM=glioblastoma multiforme; NSCLC=non-small cell lung cancer; SCLC=small cell lung cancer; $\mathrm{SD}=$ standard deviation.

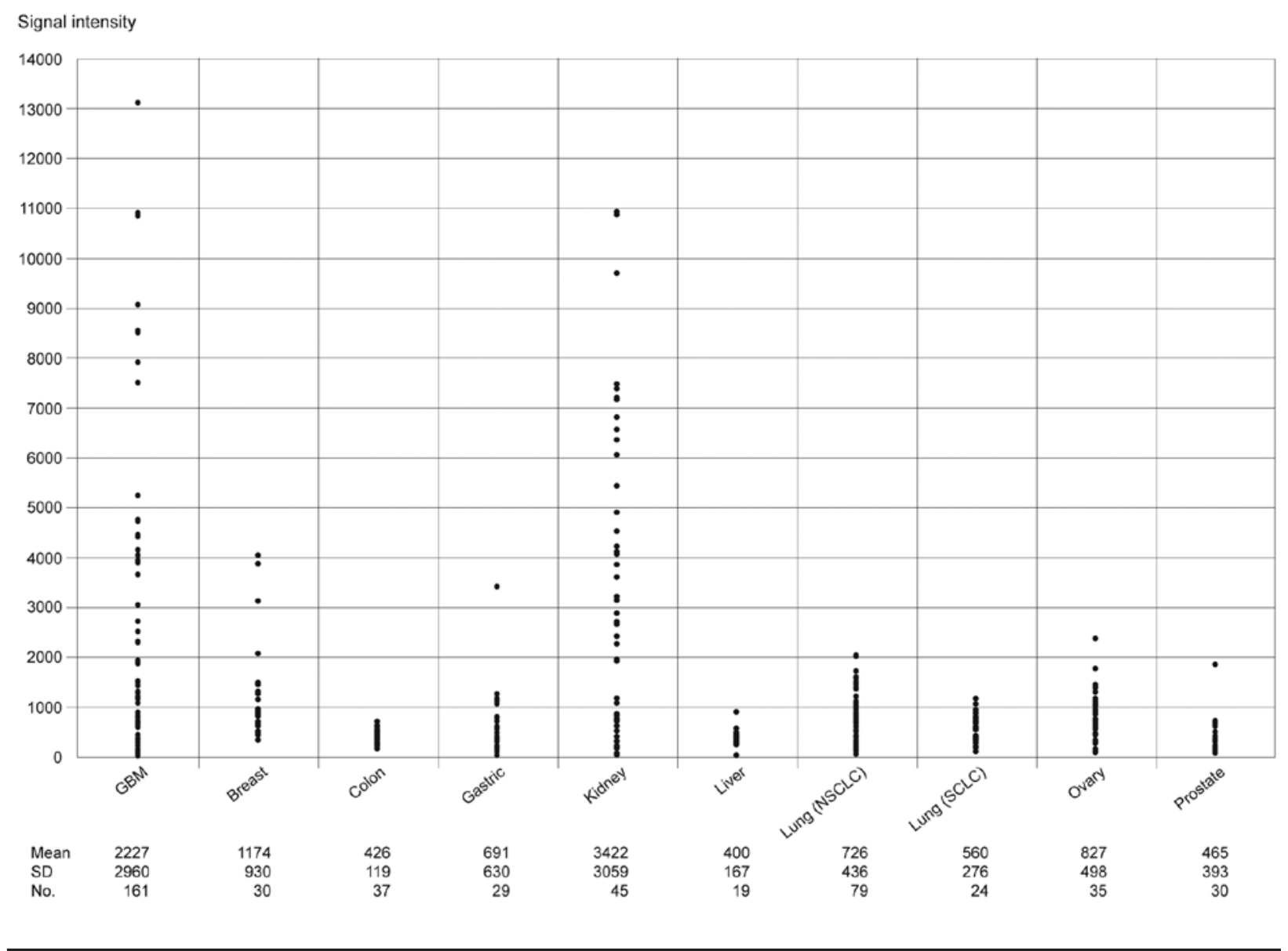


trial (the BRAIN study; AVF3708g) in GBM patients following first or second recurrence after radiotherapy and temozolomide. ${ }^{15}$ In both the bevacizumab $(n=85)$ and bevacizumab plus irinotecan $(n=82)$ cohorts, respectively, the 6-month progression-free survival (PFS) rate $(43 \%$ and $50 \%$ ), objective response rate (ORR; $28 \%$ and $38 \%$ ), and median overall survival (OS; 9.2 and 8.7 months) were compelling. Notably, ORR and 6-month PFS rate, the primary endpoints (assessed by independent radiologic review), were significantly $(P<0.0001)$ higher than the rates of $5 \%$ and $15 \%$, respectively, expected with salvage chemotherapy according to external historical controls. Other clinical benefits observed in exploratory analyses included stabilization or reduction in dose of corticosteroids for patients receiving corticosteroids at baseline, and stabilization or improvement of neurocognitive function in responders, at the time of the response, and at week 24 in those who were progression free at 24 weeks or longer. ${ }^{16}$ No unexpected toxicities outside of the well established safety profile of bevacizumab were observed in the BRAIN study. ${ }^{15}$

Given the results with bevacizumab in patients with progressive GBM following prior therapy with radiation and temozolomide, studies were initiated to evaluate bevacizumab in combination with regional radiotherapy plus temozolomide as upfront treatment for patients with newly diagnosed GBM. Safety was deemed sufficiently acceptable in pilot studies of small numbers of patients ${ }^{17,18}$ to allow expansion to larger-scale phase 2 trials.

In a noncomparative study in 70 patients with newly diagnosed GBM, ${ }^{19}$ bevacizumab in combination with radiotherapy plus temozolomide resulted in median OS and PFS of 19.6 and 13.6 months, respectively. In another nonrandomized phase 2 study, ${ }^{20}$ the current standard of care (radiotherapy plus temozolomide) was compared with $(n=25)$ and without $(n=31)$ bevacizumab in patients with newly diagnosed GBM. The 6-month PFS rate $(87 \%$ vs. $52 \%)$, median PFS (12 vs. 7 months, $P=0.0001)$, 2-year OS rate (50\% vs. $22 \%$ ), and median OS (24.0 vs. 17.5 months, $P=0.09$ ) were higher in the cohort receiving bevacizumab concurrently with standard therapy. Based on the known individual safety profile of bevacizumab, temozolomide, and radiotherapy, the investigators noted that toxicity of the combination was acceptable in the aforementioned studies. To date, there has been no full publication of controlled studies of bevacizumab in patients with recurrent GBM.

\section{AVAGLIO TRIAL}

A large, randomized, double-blind, placebocontrolled, phase 3 trial (AVAglio, BO21990, NCT00943826) evaluating the addition of bevacizumab to the current standard of care (multimodal therapy of concurrent radiotherapy plus temozolomide followed by adjuvant temozolomide) is currently recruiting patients with newly diagnosed GBM (Figure 2). It is planning to recruit a total of approximately 920 patients at approximately 140 centers worldwide. $^{21,22}$ This study aims to determine the benefit to risk profile in newly diagnosed GBM patients.

\section{Study Objectives}

The coprimary endpoints of AVAglio are to demonstrate superior OS and PFS (investigator assessment) when bevacizumab is added to temozolomide with radiotherapy followed by temozolomide. Secondary endpoints are 1-year and 2-year survival rates, PFS by independent review facility (IRF), safety, and health-related quality of life (European Organization for 
Research and Treatment of Cancer Quality of Life Questionnaire [EORTC QLQ-C30], BN20). Exploratory objectives include ORR based on adapted MacDonald criteria, response duration, OS and PFS stratified by O-6-methylguanine-DNA methyltransferase (MGMT) status, neurocognitive function using Mini Mental State Examination, corticosteroid use, signs and symptoms related to GBM, Karnofsky Performance Status, patterns of tumor progression, and biomarker analyses. The adapted MacDonald criteria that we defined take into account and clarify the evaluation of the nonenhancing component of the disease and the pseudoprogression issue, which share some concordance with recently proposed response assessment in neuro-oncology (RANO) criteria. ${ }^{23}$

\section{Key Eligibility Criteria}

Adult patients ( $\geq 18$ years of age) with newly diagnosed supratentorial GBM histologically confirmed from surgical resection or biopsy tissue are eligible for enrolment into AVAglio. Study treatment has to be initiated $>28$ and $<49$ days following the last surgical procedure and a tumor tissue block has to be available for pathology central review and analysis of MGMT status. World Health Organization (WHO) performance status has to be $\leq 2$. Corticosteroid dose has to be stable or decreasing within 5 days prior to randomization. Hematologic, hepatic, and renal function need to be adequate and blood coagulation parameters acceptable.

The main exclusion criteria include: evidence of recent intracranial hemorrhage on magnetic resonance imaging; prior chemotherapy or immunotherapy for GBM or low-grade astrocytoma; prior radiotherapy to the brain; and history of intracranial abscess within 6 months prior to randomization, serious nonhealing wound, active ulcer, or untreated bone fracture.

\section{Study Design and Therapy}

Eligible patients are randomized (1:1) to the treatment arms (bevacizumab or placebo) under double-blind conditions after central stratification for recursive partitioning analysis class as defined by EORTC/NCI-C (3, 4, and 5) and region. All patients receive local irradiation (60 Gy total dose in 2 Gy fractions 5 days per week for 6 weeks) and temozolomide $\left(75 \mathrm{mg} / \mathrm{m}^{2}\right.$ orally from the first day to the last day of radiotherapy for a maximum of 49 days) during the concurrent phase, followed by a 4-week break from temozolomide therapy starting the day after the last dose of radiotherapy, and then adjuvant temozolomide $\left(150-200 \mathrm{mg} / \mathrm{m}^{2}\right.$ daily on days 1-5 of six 4-week cycles) during the maintenance phase. Bevacizumab (10 mg/kg intravenous [IV]) or placebo is administered every 2 weeks with concurrent radiotherapy/ temozolomide, with a dose on the last day of radiotherapy/temozolomide. Following a 4-week break, bevacizumab is continued every 2 weeks concomitantly with temozolomide maintenance. Single-agent bevacizumab (15 mg/kg IV) or placebo is continued every 3 weeks during the final monotherapy phase until disease progression or unacceptable toxicity (Figure 2). Dose selection was based on previous clinical experience and activity established with this dose.

Standard methods are followed for safety monitoring/recording and treatment delay/ dose modification/discontinuation. AVAglio will meet its primary objective if one or both of the coprimary endpoints (OS and PFS) is statistically significant.

\section{CONCLUSION}

Given the dismal prognosis of newly diagnosed GBM despite treatment with the current standard of care, any treatment that 
Figure 2. AVAglio study design and treatment. $\mathrm{PD}=$ progressive disease; $\mathrm{RPA}=$ recursive partitioning analysis; $\mathrm{RT}=$ radiotherapy; $\mathrm{TMZ}=$ temozolomide.

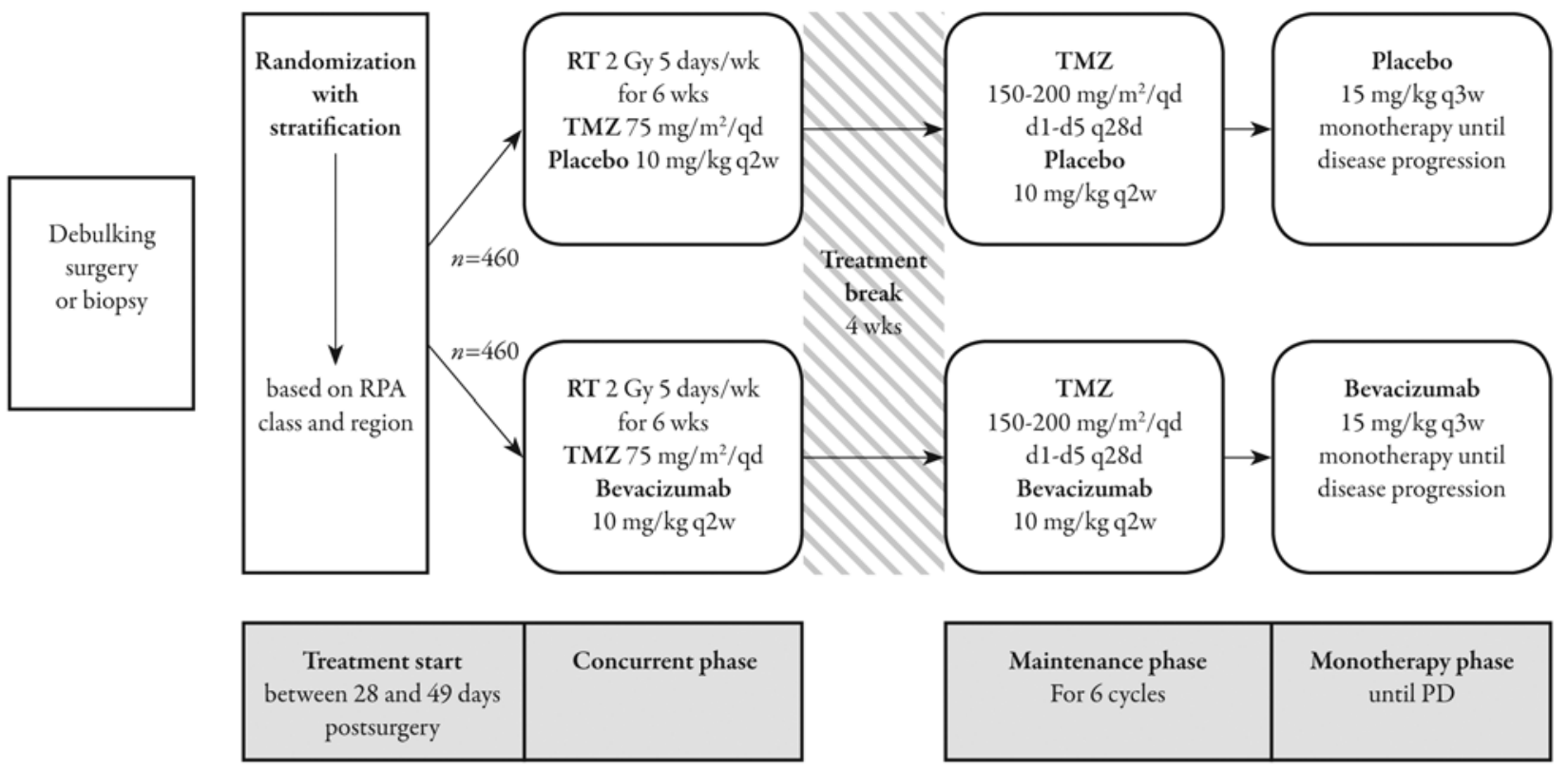

delays disease progression with positive impact on functional status and quality of life, or extends survival, would be a valuable addition. Clinical data show that bevacizumab has activity and no unexpected toxicities in patients with recurrent GBM, ${ }^{14-16}$ and several phase 2 trials indicate that combining bevacizumab with standard-of-care therapy for newly diagnosed GBM is feasible and active. ${ }^{18-20}$ Some concern has arisen that bevacizumab may promote tumor invasion by upregulation of invasion-related genes, ${ }^{24}$ although data from recent studies do not support a propensity for bevacizumab to induce diffuse tumor growth or distant spread at recurrence..$^{25,26}$

Bevacizumab has been granted US approval for use as a single agent for patients with progressive GBM following prior therapy but has not received approval for use in GBM in Europe because of the lack of controlled studies. The phase 3 AVAglio trial was therefore designed to evaluate the efficacy and safety of combining bevacizumab with standard-of-care therapy in patients with newly diagnosed GBM. This European registration trial is currently recruiting patients and results are awaited with interest. Another phase 3 study with a slightly different design conducted by the Radiation Therapy Oncology Group (NCT00884741) is ongoing in the USA in a similar patient population. ${ }^{27}$

The results of these phase 3 studies will hopefully clarify any concerns with respect to bevacizumab therapy and determine the validity of combining bevacizumab with the current standard-of-care therapy in patients with newly diagnosed GBM.

\section{ACKNOWLEDGMENTS}

OLC, TMR, and TC have received honoraria and/or financial compensation for advisory board and/or speaker activities from F. Hoffmann-La Roche Ltd. and/or Genentech, Inc.; $\mathrm{NM}, \mathrm{AZ}, \mathrm{AD}$, and $\mathrm{HP}$ are employees of F. Hoffmann-La Roche Ltd. or Genentech, 
Inc. Support for third-party writing assistance for this manuscript, furnished by Miller Medical Communications, was provided by $\mathrm{F}$. Hoffmann-La Roche Ltd. OLC is the guarantor for this article, and takes responsibility for the integrity of the work as a whole.

Open Access. This article is distributed under the terms of the Creative Commons Attribution Noncommercial License which permits any noncommercial use, distribution, and reproduction in any medium, provided the original author(s) and source are credited.

\section{REFERENCES}

1. Adamson C, Kanu OO, Mehta AI, et al. Glioblastoma mutliforme: a review of where we have been and where we are going. Expert Opin Investig Drugs. 2009;18:1061-1083.

2. Ohgaki H. Epidemiology of brain tumors. Methods Mol Biol. 2009;472:323-342.

3. Stupp R, Hegi ME, Mason WP, et al. Effects of radiotherapy with concomitant and adjuvant temozolomide versus radiotherapy alone on survival in glioblastoma in a randomised phase III study: 5-year analysis of the EORTC-NCIC trial. Lancet Oncol. 2009;10:459-466.

4. Yang JC, Haworth L, Sherry RM, et al. A randomized trial of bevacizumab, an anti-vascular endothelial growth factor antibody, for metastatic renal cancer. N Engl J Med. 2003;349:427-434.

5. Hurwitz H, Fehrenbacher L, Novotny W, et al. Bevacizumab plus irinotecan, fluorouracil, and leucovorin for metastatic colorectal cancer. N Engl J Med. 2004;350:2335-2342.

6. Sandler A, Gray R, Perry MC, et al. Paclitaxelcarboplatin alone or with bevacizumab for non-small-cell lung cancer. $\mathrm{N}$ Engl J Med. 2006;355:2542-2550.

7. Miller K, Wang M, Gralow J, et al. Paclitaxel plus bevacizumab versus paclitaxel alone for metastatic breast cancer. N Engl J Med. 2007;357:2666-2676.

8. Ferrara N. Vascular endothelial growth factor: basic science and clinical progress. Endocr Rev. 2004;25:581-611.
9. Hicklin DJ, Ellis LM. Role of the vascular endothelial growth factor pathway in tumor growth and angiogenesis. J Clin Oncol. 2005;23:1011-1127.

10. Gerber HP, Ferrara N. Pharmacology and pharmacodynamics of bevacizumab as monotherapy or in combination with cytotoxic therapy in preclinical studies. Cancer Res. 2005;65:671-680.

11. Mathieu V, De Neve N, Le Mercier $M$, et al. Combining bevacizumab with temozolomide increases the antitumor efficacy of temozolomide in a human glioblastoma orthotopic xenograft model. Neoplasia. 2008;10:1383-1392.

12. Lee CG, Heijn M, di Tomaso E, et al. Anti-vascular endothelial growth factor treatment augments tumour radiation response under normoxic or hypoxic conditions. Cancer Res. 2000;60:5565-5570.

13. Vredenburgh JJ, Desjardins A, Herndon JE 2nd, et al. Bevacizumab plus irinotecan in recurrent glioblastoma multiforme. J Clin Oncol. 2007;25:4722-4729.

14. Kreisl TN, Kim L, Moore K, et al. Phase II trial of single-agent bevacizumab followed by bevacizumab plus irinotecan at tumor progression in recurrent glioblastoma. J Clin Oncol. 2009;27:740-745.

15. Friedman HS, Prados MD, Wen PY, et al. Bevacizumab alone and in combination with irinotecan in recurrent glioblastoma. J Clin Oncol. 2009;27:4733-4740.

16. Vredenburgh JJ, Wefel J, Coughesy $\mathrm{T}$, et al. Clinical assessment of corticosteroid use and neurocognitive function in patients with glioblastoma at first or second relapse treated with bevacizumab in the BRAIN study. Eur J Cancer Suppl. 2009;7:495 (abstract O-8701).

17. Lai A, Filka E, McGibbon B, et al. Phase II pilot study of bevacizumab in combination with temozolomide and regional radiation therapy for up-front treatment of patients with newly diagnosed glioblastoma multiforme: interim analysis of safety and tolerability. Int $\mathrm{J}$ Radiat Oncol Biol Phys. 2008;71:1372-1380.

18. Narayana A, Golfinos JG, Fischer I, et al. Feasibility of using bevacizumab with radiation therapy and temozolomide in newly diagnosed high-grade glioma. Int J Radiat Oncol Biol Phys. 2008;72:383-389. 
19. Lai A, Tran A Nghiemphu PL, et al. Phase II trial of bevacizumab plus temozolomide during and after radiation therapy for patients with newly diagnosed glioblastoma multiforme. J Clin Oncol. 2011;29:142-148.

20. Gruber ML, Raza S, Gruber D, Narayana A. Bevacizumab in combination with radiotherapy plus concomitant and adjuvant temozolomide for newly diagnosed glioblastoma: update progressionfree survival, overall survival, and toxicity. J Clin Oncol. 2009;27(Suppl):15s (abstract 2017).

21. Anon. A study of Avastin (bevacizumab) in combination with temozolomide and radiotherapy in patients with newly diagnosed glioblastoma. Available at: http://clinicaltrials.gov/ct2/show/ NCT00943826. Accessed July 14, 2010.

22. Chinot $\mathrm{O}$, de La Motte Rouge $\mathrm{T}$, Moore N, Zeaiter A. Addition of bevacizumab to the multi-modality standard care in patients with newly diagnosed glioblastoma: a phase III trial. Poster presented at: 34th ESMO Multidisciplinary Congress, September 20-24, 2009; Berlin, Germany.
23. Wen PY, Macdonald DR, Reardon DA, et al. Updated response assessment criteria for high-grade gliomas: response assessment in neuro-oncology working group. J Clin Oncol. 2010;28:1963-1972.

24. Lucio-Eterovic AK, Piao Y, de Groot JF. Mediators of glioblastoma resistance and invasion during antivascular endothelial growth factor therapy. Clin Cancer Res. 2009;15:4589-4599.

25. Pope WB, Xia Q, Paton VE, et al. Patterns of progression in patients with recurrent glioblastoma treated with bevacizumab. Neurology. 2011;76:432-437.

26. Wick W, Wick A, Weiler M, Weller M. Patterns of progression in malignant glioma following antiVEGF therapy: perceptions and evidence. Curr Neurol Neurosci Rep. In press.

27. Anon. Temozolomide and radiation therapy with or without bevacizumab in treating patients with newly diagnosed glioblastoma or gliosarcoma. Available at: http://clinicaltrials.gov/ct2/show/ NCT00884741. Accessed July 14, 2010. 